\title{
PORTABLE MICROFLUIDIC OPTOELECTRONIC DEVICE FOR SALIVARY DIAGNOSTICS OF STOMACH CANCER
}

\author{
Yael Zilberman and Sameer R. Sonkusale \\ Nano Lab, Tufts University, Medford, MA, USA
}

\begin{abstract}
Stomach cancer is the second most common cause of cancerrelated deaths in the world and its primary identified cause is infection by a gram-negative bacterium Helicobacter pylori. These bacteria survive in a highly acidic stomach environment by converting urea into bicarbonate and ammonia that decreases acidity. We present a microfluidic optoelectronic sensor for detection of ammonia and carbon dioxide in saliva, for early diagnosis of stomach cancer. The sensor is composed of an array of microwells embedded in a microfluidic device. The microwells are filled with polymeric ion-exchange microbeads doped with organic dyes. Instead of monitoring response at a single wavelength, the optoelectronic monitoring of this unique sensor platform is done over broad wavelength spectrum providing excellent sensitivity and specificity to different concentrations of carbon dioxide and ammonia dissolved in saliva with a detection limit in a ppm range. Our ultimate goal is to develop a noninvasive, fast and portable diagnostic platform that can be used for early screening of gastric cancer, as an alternative to currently existing diagnostic procedures.
\end{abstract}

\section{INTRODUCTION}

Gastric cancer is one of the most common causes of death from cancer worldwide. In the United States, the estimated new cases from gastric cancer in 2013 are 21,600, including 10,990 deaths [1]. Because of unspecific clinical symptoms and not welldefined risk factors, the diagnosis is often delayed, leading to low survival rate. In Europe, the 5-year survival is below $25 \%$, and the situation is even worse in the United States. Earlier diagnosis substantially improves the rate of survival: $95 \%$ of patients with cancerous growth, which is confined to the inner stomach lining, will survive longer than 5 years [2]. The primary marker for early identification of stomach cancer is infection with gram-negative bacterium Helicobacter pylori [3]. It has been shown that when the bacterium is eliminated in treated patients at early stage of gastric cancer, the risk of developing a second gastric cancer decreases by two-thirds [1]. To survive in the harsh, acidic environment of the stomach, $H$. pylori secretes the enzyme urease that converts urea into bicarbonate and ammonia (neutralizing the acidic environment locally).

Gastric cancer diagnosis is normally done by endoscopy with biopsy and histopathological evaluation, which provides a high diagnostic accuracy of 95-99\% [4]. However, the invasive and relentless character of this procedure makes it less suitable for fast screening. In addition, the method is relatively expensive and requires highly skilled medical personnel. Moreover, some early gastric cancers provide very little optical contrast with the surrounding tissues and could be missed during endoscopic examination. Endoscopy with biopsy can be also used for the identification of $H$. pylori infection, when combined with a rapid urease test and microbial culture. Urease can be also detected in serum, using enzyme-linked immunosorbent assay (ELISA), but collecting blood is required in this case [5]. Another approach is breath analysis: isotopically-labeled urea is orally ingested by the patient and the breath sample is subsequently analyzed by mass spectrometry, to test for (isotopically labeled) nitrogen and carbon. This approach is noninvasive, but requires expensive equipment.
Recently, saliva has been proposed as a promising diagnostic fluid for early screening of gastric cancer. Salivary diagnostics is much more convenient than biopsy or blood test: sample collection is simple, non-invasive and, therefore, not painful [6-8]. With respect to the gastric cancer screening, saliva is commonly analyzed for the presence of abnormal genetic material, or of specific biomarkers, which are detected by analyte-specific interactions using immunoassay arrays [9]. While these techniques are effective in early detection of gastric cancer, they are rather inappropriate for fast and high throughput screening, particularly outside of medical facilities. In addition, immunoassays are analyte-specific and, therefore, limited to measurement of just a few, among many, biomarkers.

It has been shown recently by means of gas chromatography/mass spectrometry (GC-MS) that, just as in the case of exhaled breath, there are a variety of volatile compounds in saliva [8]. Some volatile compounds found in exhaled breath, such as ammonia and carbon dioxide, are related to gastric cancer [10]. It is expected that volatile compounds exhaled from the oral cavity should be present both in the exhaled breath and in saliva, suggesting that salivary ammonia and carbon dioxide analysis has a potential for non-invasive early screening of gastric cancer. However, GC-MS is time consuming, not portable and requires complex and expensive equipment, which makes it less suitable for fast and portable screening.

An alternative approach is implementation of gas-phase crossreactive sensor arrays known as "electronic noses", using chemiresistors or field-effect transistors that provide an ensemble electrical response [11]. The research in this field is quite advanced and this concept has been already implemented for detection of numerous volatiles related to various types of cancer, including gastric cancer [11]. Another approach is based on the use of optical arrays, composed of various organic dyes which sensitive to a particular group (or groups) of volatiles, providing an ensemble optical response, which is used for analyte classification [12].

So far, cross-reactive sensors have not been implemented for detection of ammonia and carbon dioxide in saliva. There are some important advantages in analyzing liquid samples. First, collected samples can be easily stored, since only tiny volumes are required. Second, salivary materials concentrations are higher than those in breath samples, where the exhaled materials are diluted by air. Finally, it is simpler to prevent contamination from the environment. We have developed a portable optoelectronic device based on a microfluidic platform and the sensing material is composed of three different organic dyes encapsulated in ion exchange resin beads. Conventionally, optical sensors rely on a response at a single wavelength, or a narrow range. Our crossreactive sensor reads the entire visible spectrum and therefore, could extract unique multi-dimensional signatures based on colorimetric or fluorescent response of various dyes at different wavelengths. Such readouts from the microsensor array will capture multiple physical and chemical interactions between the dissolved gases (ammonia and carbon dioxide) and the composite sensing material and, therefore, are expected to be more sensitive and selective than other conventional techniques. Here, we demonstrate the excellent performance of our miniature optical sensor for analyzing ppm-level concentrations of ammonia and carbon dioxide dissolved in human saliva. 


\section{SENSOR FABRICATION Sensing Material}

1. Ion pair preparation: The procedure of ion pair preparation was based on our previously published work [13]. Briefly, the $\mathrm{pH}-$ sensitive dyes were either cresol red or pyranine (Aldrich) and tetraoctylammonium hydroxide $(\mathrm{TOAOH})$ was used as an ionpairing agent. TOAOH was prepared from tetraoctylammonium bromide (TOABr) (Aldrich) and silver oxide (Aldrich). The dye containing solution (S1) was prepared by dissolving either cresol red or pyranine in $0.1 \mathrm{M} \mathrm{NaOH}$. TOABr solution in organic phase (S2) was prepared by dissolving TOABr in toluene. Solutions S1 and $\mathrm{S} 2$ were mixed and stirred and the organic phase was removed and washed with $0.1 \mathrm{M} \mathrm{NaOH}$. The resulting organic phase contained the pure ion pair of either [cresol red-/TOA+] or [pyranine-/ $\mathrm{TOA}+$ ] dissolved in toluene.

2. Ion pair encapsulation: The ion pair consisting of an organic quaternary ammonium cation and a $\mathrm{pH}$ indicator dye anion was encapsulated within ion exchange resin beads according to the following procedure: $560 \mathrm{mg}$ of anion exchange resin microbeads (Dowex ${ }^{\circledR}$ chloride form, 200-400 mesh, Sigma-Aldrich), $1 \mathrm{~mL}$ of TOAOH, $1 \mathrm{~mL}$ of ion pair solution in toluene and $2 \mathrm{~mL}$ of toluene were mixed and homogenized by stirring for $1 \mathrm{~h}$. The resulted suspension was dried in an oven at $50^{\circ} \mathrm{C}$ overnight in order to evaporate the toluene. The dry powder was dissolved in $5 \mathrm{ml}$ ethanol. Eventually, $5 \mathrm{~mL}$ water was added to the dye-doped microbeads suspension before the microbeads deposition into the wells of microfluidic device.

3. $\mathrm{Zn}$ (TPP) encapsulation: The dye solution was prepared by dissolving $22.5 \mathrm{mg}$ of 5,10,15,20-Tetraphenyl-21H,23H-porphine zinc (Zn(TPP)) dye (Sigma-Aldrich) in $1 \mathrm{ml}$ of ethanol (> $99.5 \%$, Sigma-Aldrich), followed by addition of $4 \mathrm{ml}$ of deionized water. Subsequently, $560 \mathrm{mg}$ of cation exchange resin microbeads powder (Dowex ${ }^{\circledR}$ 50WX4 hydrogen form, 200-400 mesh, Sigma-Aldrich) and $5 \mathrm{ml}$ of deionized water were added to the dye solution and stirred for $5 \mathrm{~h}$ at room temperature, in order to create ionic bonding between the $\mathrm{Zn}$ (TPP) dye and the beads.

\section{Saliva Collection}

A volunteer was instructed to expectorate every $30 \mathrm{~s}$ into a 50 $\mathrm{mL}$ glass beaker after being asked to stay $30 \mathrm{~min}$ without food and liquid. A total of $10 \mathrm{~mL}$ of whole saliva was collected. The saliva was diluted ten-fold by adding deionized water under continuous stirring using a Teflon magnetic stirrer bar. The saliva sample was analyzed immediately after the collection.

\section{Microfluidic Device}

1. Master fabrication: A mask with desired master features was previously fabricated. $1 \mathrm{ml}$ of SU-8 2100 was spin-coated on the $4^{\prime \prime}$ wafer, followed by soft bake at $65^{\circ} \mathrm{C}$ for $30 \mathrm{~min}$ and subsequently at $95^{\circ} \mathrm{C}$ for $90 \mathrm{~min}$. The wafer was then placed into the OAI Model 204 mask aligner and exposed to 365nm light with $20 \mathrm{~mW} / \mathrm{cm}^{2}$ energy dose for $30 \mathrm{sec}$, followed by post exposure bake at $65^{\circ} \mathrm{C}$ for $1 \mathrm{~min}$ and subsequently at $95^{\circ} \mathrm{C}$ for $20 \mathrm{~min}$. The wafer was developed in the SU-8 developer for $\sim 25-30 \mathrm{~min}$. After the development, the wafer was rinsed with isopropanol and dried using a nitrogen gun.

2. PDMS mold fabrication: The base/curing agent ratio (of the PDMS pre-polymer components) was 10:1. The prepared PDMS mixtures were first placed in a vacuum desiccator for $30 \mathrm{~min}$ (for degassing) and subsequently poured on top of the master, followed by vacuum degassing for $30 \mathrm{~min}$. The master was then placed inside an oven at $70^{\circ} \mathrm{C}$ for $4 \mathrm{~h}$ to cure the device layer. The PDMS mold was cut around the edge of the master wafer and peeled up. Fluid inlet and outlet holes of the PDMS device were punched using a 16-gauge needle before placing it into the RIE chamber. The March CS-1701F Reactive Ion Etcher was used $(50 \mathrm{mT})$. Oxygen was flowed for $30 \mathrm{sec}$ at $50 \mathrm{Watt} \mathrm{RF}$ power. Immediately after removing from the RIE chamber, the PDMS molds were pressed gently, ensuring that a strong bond is formed.

\section{EXPERIMENTAL SETUP}

Fig. 1 shows the experimental setup complete with flow system and optical readout. White LED is used as a light source. Light transmitted through the sensor array is guided by a $400 \mu \mathrm{m}$ premium fiber through a collimating lens connected to a portable spectrometer (USB650, Ocean Optics). The spectrometer captures the entire spectrum instead of conventional readout approach using optical filters that measures response at a single wavelength.

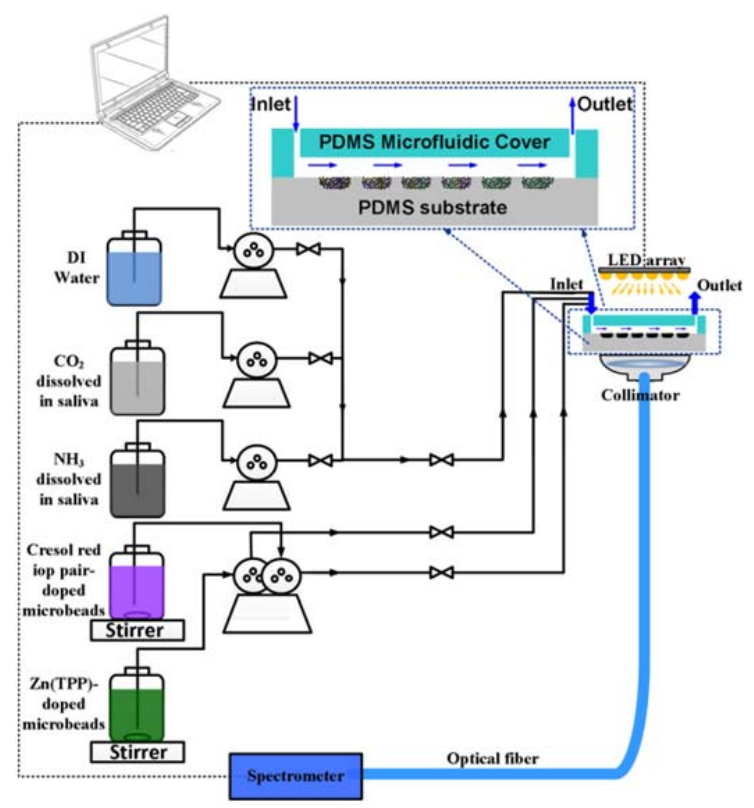

Figure 1: Schematic representation of the microfluidic sensor device, an array of microwells embedded in a microfluidic device. Optoelectronic setup and flow system are also shown.

\section{DYE-DOPED MICROBEADS DEPOSITION}

Ion exchange resin microbeads $(\sim 40-80 \mu \mathrm{m})$ doped with organic dye (Zn(TPP) and either cresol red/quaternary ammonium or pyranine/quaternary ammonium cation ion pair) were deposited into the microwells $(500 \mu \mathrm{m}$ diameter and depth) of the microfluidic device by circulating the microbeads suspension through the device using a peristaltic pump (Fig. 1 and 2). The device has three inlets and three outlets. Two inlets and outlets are used for deposition (for two different types of resin microbeads doped with dyes) and the ones in the middle are used for the device exposure to analyte solutions. The cation $\mathrm{Zn}(\mathrm{TPP})$ dye is encapsulated within cation exchange resin beads, while the anion $\mathrm{pH}$ dye ion pair is encapsulated within anion exchange resin beads. The opposite charge of two types of the microbeads makes their simultaneous deposition using a single microfluidic channel challenging, since the microbeads coagulate strongly because of electrostatic interactions. To resolve this issue, the deposition of two types of microbeads was done using separate channels. Figure 2 shows the microfluidic device embedding the array with about a half of microwells filled with microbeads doped with $\mathrm{pH}$ dye/quaternary ammonium cation ion pair and another half with microbeads doped with $\mathrm{Zn}(\mathrm{TPP})$. 

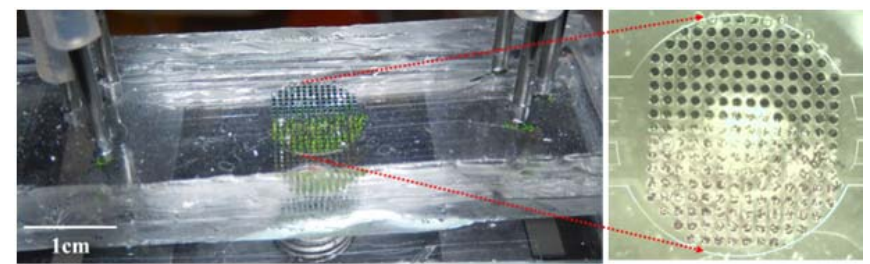

Figure 2: The array $(d=10 \mathrm{~mm}$, each microwell is $500 \mu \mathrm{m})$ is embedded in the microfluidic device with inlet and outlet channels. The array microwells are filled with microbeads doped with pyranine or cresol red-based ion pairs and with Zn(TPP).

\section{SENSOR PERFORMANCE}

In the following discussion, $100 \%$ concentration of dissolved $\mathrm{CO}_{2}$ and $\mathrm{NH}_{3}$ refers to $150 \mathrm{ppm}$ and $18 \mathrm{ppm}$, respectively. Figure 3 shows transmission spectra recorded under the sensor exposure to the mixture of $90 \% \mathrm{CO}_{2}(135 \mathrm{ppm})$ and $10 \% \mathrm{NH}_{3}$ (1.8ppm) dissolved in DI water (Fig. 3a) and human saliva (Fig. 3b). White LED was used as a light source and the irradiation intensity transmitted through the array was measured in the visible spectrum (440-680nm) using a portable spectrometer (schematic representation of the optical setup is shown in Fig. 1). All spectra were first normalized to the maximal transmission intensity and the reference spectrum obtained with deionized water was subsequently subtracted, i.e. $\Delta \mathrm{T}=0$ means no response and higher $\Delta \mathrm{T}$ (absolute) magnitude indicates higher response.

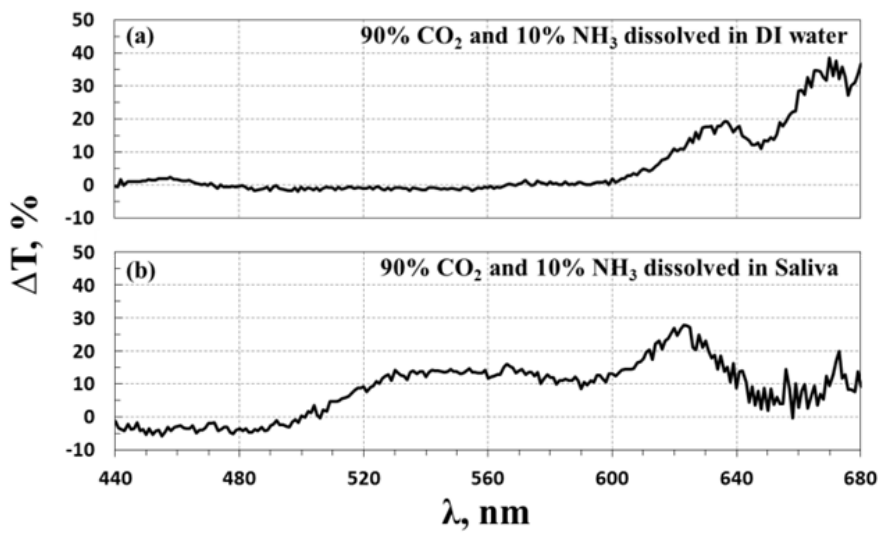

Figure 3: Transmittance spectra recorded under the sensor exposure to mixture of 130ppm $\mathrm{CO}_{2}$ and 1.8ppm $\mathrm{NH}_{3}$ dissolved in (a) DI water and (b) diluted saliva.

There are clear changes in transmittance upon the exposure to the mixture of $\mathrm{CO}_{2}$ and $\mathrm{NH}_{3}$ dissolved in either DI water (Fig. 3a) or diluted human saliva (Fig. 3b). It can be seen that the response magnitude and the shape of the obtained spectra differ significantly depending on the media (DI water or diluted saliva). There are spectral shifts in different ranges of the spectrum. In the case of DI water the shift was positive for $440-470 \mathrm{~nm}$ and $600-680 \mathrm{~nm}$, while no response was recorded for $470-600 \mathrm{~nm}$. In the case of saliva the shift was positive for $500-680 \mathrm{~nm}$ and negative for $440-500 \mathrm{~nm}$. In principle, such "signatures" over a broad optical spectrum can be used to distinguish dissolved $\mathrm{CO}_{2}$ and $\mathrm{NH}_{3}$ from other analytes that present in human saliva, as well as in other complex environments.
The observed spectral shifts correspond to the change in the dye color. The color of $\mathrm{Zn}$ (TPP) changes from green to purple under the exposure to dissolved $\mathrm{NH}_{3}$. On the other hand, the colors of the pyranine-based ion pair and of the cresol red-based ion pair change under the exposure to dissolved $\mathrm{CO}_{2}$ from shiny yellow to transparent and from purple to yellow, respectively. All these changes correspond to increase or decrease in the transmission spectrum. This observation emphasizes the advantage of the sensing strategy that employs hyperspectral imaging by analyzing the entire visible spectrum instead of focusing on a specific wavelength or a narrow range, as it is often done with optical sensors.

Figure 4 shows representative examples of the sensor response to mixtures of $\mathrm{CO}_{2}$ and $\mathrm{NH}_{3}$ with different concentrations, dissolved in either DI water (Fig. 4a) or diluted human saliva (Fig. $4 \mathrm{~b})$, at two wavelengths $(640 \mathrm{~nm}$ and $680 \mathrm{~nm})$. The response is shown in terms of the measured intensity (I) normalized to the baseline intensity $\left(\mathrm{I}_{\mathrm{b}}\right.$, recorded when the device is exposed to distilled water), as a function of concentrations (maximum $(100 \%)$ concentrations of $\mathrm{CO}_{2}$ and $\mathrm{NH}_{3}$ are $150 \mathrm{ppm}$ and $18 \mathrm{ppm}$, respectively). The sensor contained an array composed of microbeads doped with a mixture of microbeads doped with $\mathrm{Zn}(\mathrm{TPP})$ and pyranine- or cresol red-based ion pair.

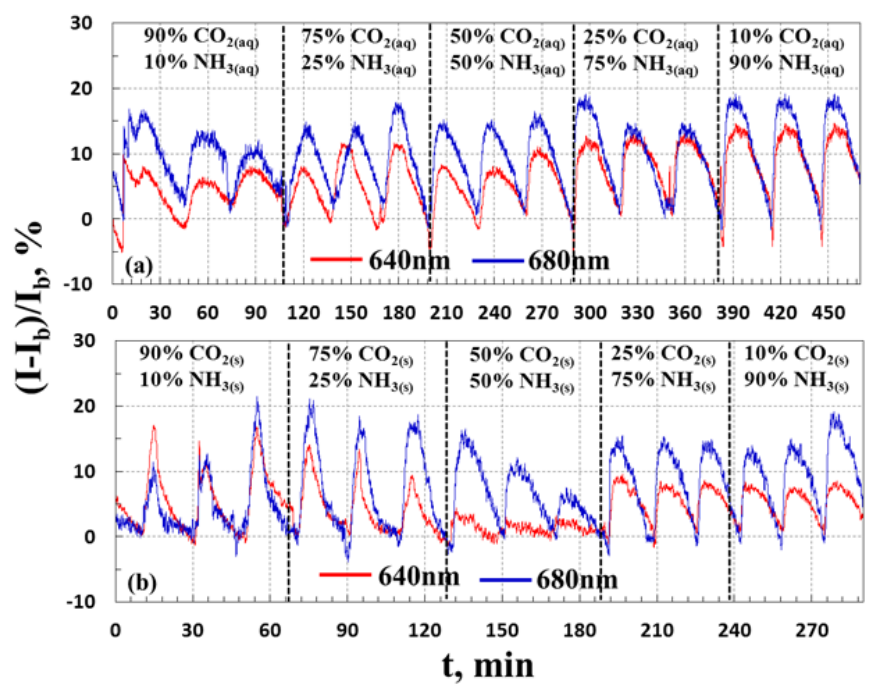

Figure 4: Sensor response to different concentrations of mixture contained $\mathrm{CO}_{2}$ and $\mathrm{NH}_{3}$ dissolved in (a) DI water and (b) diluted saliva at 640nm (red colour) and 680nm (blue color).

The response magnitude is quite different for DI water and human saliva, while the response shape is similar for most of concentrations. However, the shape of the responses for the mixture of $90 \% \mathrm{CO}_{2}$ and $10 \% \mathrm{NH}_{3}$ and $75 \% \mathrm{CO}_{2}$ and $25 \% \mathrm{NH}_{3}$ in human saliva is very different. This indicates that the response of ion pairs based on halochromic dyes (cresol red and pyranine) to $\mathrm{CO}_{2}$ in the presence of $\mathrm{NH}_{3}$ is more pronounced in human saliva than in DI water. For DI water the sensor response is dominated by the response to $\mathrm{NH}_{3}$ even at its low concentrations $(10 \%$ and $25 \%$, which corresponds to $1.8 \mathrm{ppm}$ and $4.5 \mathrm{ppm}$ ).

Figure 5 summarizes the results obtained in the entire (visible) spectrum, in terms of the response magnitude in the twodimensional plane of wavelength vs. concentration. Evidently, the sensor is sensitive to different concentrations of diluted saliva (Fig. $5 \mathrm{a}, 100 \%$ corresponds to the saliva sample ten-fold diluted by DI 
water) and to different mixtures of $\mathrm{CO} 2$ and $\mathrm{NH} 3$ dissolved in DI water (Fig. 5b) and human saliva (Fig. 5c).

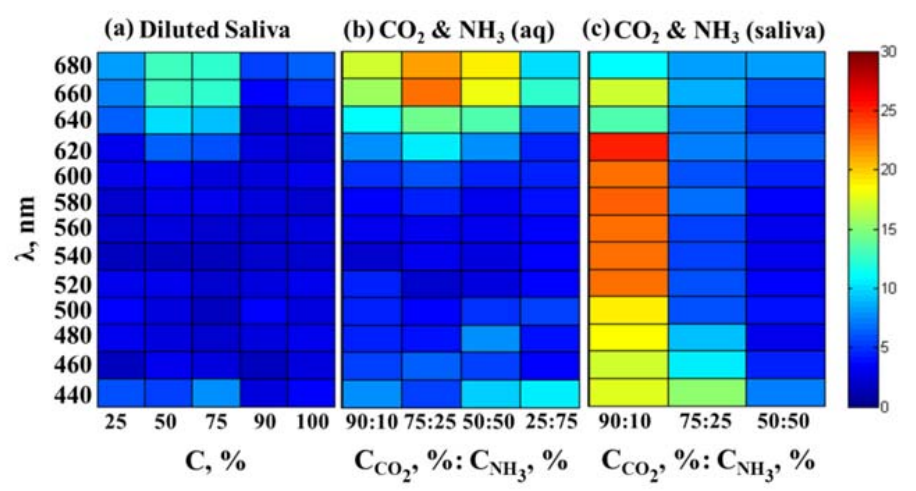

Figure 5: Sensor response magnitude at a range of wavelengths for diluted saliva (a) and for the mixture of $\mathrm{CO}_{2}$ and $\mathrm{NH}_{3}$ dissolved in deionized water (b) and diluted human saliva (c).

The saliva of healthy person contains low concentrations of $\mathrm{CO}_{2}$ (ppm level) and $\mathrm{NH}_{3}$ (sub-ppm level) $[8,10]$. However, in the presence of the $H$. pylori infection in the stomach, concentrations of $\mathrm{CO}_{2}$ and $\mathrm{NH}_{3}$ in saliva are significantly higher. The obtained "fingerprints" differ sufficiently in order to discriminate between diluted saliva, $\mathrm{CO}_{2}$ and $\mathrm{NH}_{3}$ dissolved in DI water, and $\mathrm{CO}_{2}$ and $\mathrm{NH}_{3}$ dissolved in human saliva. These results indicate feasibility of this sensor platform for early diagnostics of gastric cancer sensor based on its ability to distinguish between the saliva of a healthy person and the saliva with elevated concentrations of $\mathrm{CO}_{2}$ and $\mathrm{NH}_{3}$ (H. pylori infection).

\section{CONCLUSIONS}

We have developed a novel optoelectronic sensor for crossreactive detection of $\mathrm{CO}_{2}$ and $\mathrm{NH}_{3}$ dissolved in human saliva. The sensor is based on a microfluidic platform and contains a crossreactive array composed of metalloporphyrin dye and halochromic dye/quaternary ammonium ion pair ionically bonded to ionexchange polymeric microbeads. The use of the composite sensing material and broad spectrum optical readout offers important advantages for sensitive detection of dissolved $\mathrm{CO}_{2}$ and $\mathrm{NH}_{3}$ in complex environments, directly in liquid samples and without the use of any separating membrane. Strong ionic interactions between the ion-exchange microbeads and the dyes prevent the wash out of the sensing material.

The procedure for composite microbeads deposition into the sensing device is simple and robust and allows easy incorporation of virtually any combination of organic dyes, providing a route for cross-reactive sensing. Due to the flexible optical setup, the sensor can be easily modified for a variety of applications, where portable detection of dissolved volatiles in small liquid samples is required. The overall platform is low cost and portable. The sensor is sensitive to ppm levels of dissolved $\mathrm{CO}_{2}$ and $\mathrm{NH}_{3}$ in human saliva. This provides a potential for a variety of medical diagnostic applications, for early gastric cancer diagnosis in particular.

High surface-to-volume fraction of the composite microbeads is expected to provide relatively fast response times, but the chemical interaction between the dissolved $\mathrm{CO}_{2}$ and $\mathrm{NH}_{3}$ and the organic dyes maybe a limiting step. Residence time inside the microfluidic devices yet to be optimized. A trade-off between fast response times and sensor array stability and durability is yet to be identified. To achieve a truly cross-reactive approach, a large number of various organic dyes should be implemented. Possible interference with other chemical compounds should be also carefully investigated.

\section{ACKNOWLEDGMENTS}

The authors would like to acknowledge the partial support from National Science Foundation under grant EFRI: 1240443. The device fabrication was performed at the Tufts Micro and Nanofabrication facility.

\section{REFERENCES}

[1] National Cancer Institute, www.cancer.gov.

[2] K. D. Crew and A. I. Neugut, "Epidemiology of Gastric Cancer," World Journal of Gastroenterology, 12, 354 (2006).

[3] N. Uemura, S. Kamoto, S. Amamoto, N. Atsumura, S. Amaguchi, M. Amakido, K. Aniyama, N. Sasaki and R. J. Schlemper," Helicobacter Pylori Infection and the Development of Gastric Cancer", The New England Journal of Medicine, 345, 784 (2001).

[4] C. P. Dooley, A. W. Larson, N. H. Stace, I. G. Renner, J. E. Valenzuela, J. Eliasoph, P. M. Colletti, J. M. Halls and J. M. Weiner, "Double-Contrast Barium Meal and Upper Gastrointestinal Endoscopy: A Comparative Study”, Annals of Internal Medicine, 101, 538 (1984)

[5] J. Parsonnet, I. M. Samloff, L. M. Nelson, N. Orentreich, J. H. Vogelman and G. D. Friedman, "Helicobacter Pylori, Pepsinogen, and Risk for Gastric Adenocarcinoma," Cancer Epidemiology, Biomarkers \& Prevention, 2, 461 (1993).

[6] C. F. Streckfus and L. R. Bigler, "Saliva as a Diagnostic Fluid," Oral Diseases, 8, 69 (2002).

[7] H. J. Martin, S. Riazanskaia and C. L. Thomas, "Sampling and Characterisation of Volatile Organic Compound Profiles in Human Saliva using a Polydimethylsiloxane Coupon Placed within the Oral Cavity," Analyst, 137, 3627 (2012).

[8] H. A. Soini, I. Klouckova, D. Wiesler, E. Oberzaucher, K. Grammer, S. J. Dixon, Y. Xu, R. G. Brereton, D. J. Penn and M. V. Novotny, "Analysis of Volatile Organic Compounds in Human Saliva by a Static Sorptive Extraction Method and Gas Chromatography-Mass Spectrometry," J Chem Ecol, 36, 1035 (2010).

[9] W. Tan, L. Sabet, Y. Li, T. Yu, P. R. Klokkevold, D. T. Wong, C. M. Ho, "Optical Protein Sensor for Detecting Cancer Markers in Saliva," Biosensors and Bioelectronics, 24, 266 (2008).

[10] B. Timmer, W. Olthuis, A.V.D. Berg, "Ammonia Sensors and their Applications - a Review", Sensors and Actuators B, 107, 666 (2005).

[11] Z. Q. Xu, Y. Y. Broza, R. Ionsecu, U. Tisch, L. Ding, H. Liu, Q. Song, Y. Y. Pan, F. X. Xiong, K. S. Gu, G. P. Sun, Z. D. Chen, M. Leja, H. Haick, "A Nanomaterial-based Breath Test for Distinguishing Gastric Cancer from Benign Gastric Conditions," British Journal of Cancer, 108, 941 (2013).

[12] N. A. Rakow and K. S. Suslick, "A Colorimetric Sensor Array for Odour Visualization," Letters to Nature, 406, 710 (2000).

[13] Y. Zilberman, S. K. Ameri, S. R. Sonkusale, "Microfluidic Optoelectronic Sensor based on a Composite Halochromic material for Dissolved Carbon Dioxide Detection", Sensors and Actuators B, 194, 404, (2014).

\section{CONTACT}

* S. R. Sonkusale, tel: +1-617-627-5113; sameer@ece.tufts.edu 